\title{
Yoga for Improving Mental Health during COVID-19 Pandemic: A Review
}

\author{
Dhananjoy Shaw ${ }^{1}$, Chitra Chand $^{2}$
}

\begin{abstract}
Background: Novel coronavirus disease 2019 (COVID-19), a deadly viral disease is caused by severe acute respiratory syndrome coronavirus-2 (SARS-CoV-2) that has caused over a million deaths globally and still counting. Many countries are observing a nationwide lockdown since November 2019 that varied from time to time and country to country to decrease its spread and the governments are urging the people to follow safety guidelines such as face and nose masking, different hygienic habits, sanitization, repeated handwashing, avoiding crowd, not spitting in the public place, not using the tobacco products, and social distancing, etc. that may cause loneliness, mental distress, anxiety, and depression. Method : Electronic database PubMed was searched from 1 December 2019 to 25 November 2020 using the keywords "coronavirus disease, COVID-19, yoga, meditation, pranayama, mindfulness, and mental health." Out of 95 studies, 23 were selected for the review.

Result: There are no studies published to evaluate the effect of yoga on mental health during the spread of COVID-19 pandemic on people. However, the studies published on the effect of yoga and meditation for improving mental health are of great importance to deal with such a challenging COVID-19 pandemic period.

Conclusion: Yoga and meditation can be used as a preventive and coping measure to combat COVID-19 and to reduce the harmful psychological effects due to COVID-19.

Keywords: COVID-19, Meditation, Mindfulness, Pranayama, Yoga.

Eastern Journal of Psychiatry (2020): 10.5005/jp-journals-11001-0004
\end{abstract}

\section{INTRODUCTION}

Novel coronavirus 2019 (COVID-19), a pandemic caused by SARSCoV-2 that has spread globally emerged as a group of unexplained pneumonia cases in Wuhan, China in December, 2019., ${ }^{1,2}$ COVID-19 was declared a Public Health Emergency of International Concern by WHO on 30 January $2020 .^{3}$

Till date there are no specific drugs, treatment protocol, and vaccination for the coronavirus disease, and the treatment includes isolation and symptomatic management. ${ }^{2}$ The preventive measures to stop the transmission of the disease are social distancing, frequent hand washing, and quarantine in some cases. ${ }^{1,4}$ Previous studies on SARS and Ebola outbreak in 2003 and 2014, respectively, reported the unfavorable psychological effects of quarantine like post-traumatic stress symptoms, confusion, and anger, and people with pre-existing anxiety and depression may show worse psychological symptoms due to quarantine and social distancing. ${ }^{1}$ The easy access to different types of news, false claims, and misinformation propagated through print media, news channels, and social media on COVID-19 can enhance the aforementioned symptoms. ${ }^{5,6}$ Poor mental conditions such as depression and stress, also elevates the possibility of acute respiratory infections. ${ }^{7}$

Due to work from home, the electronic and distancing means of communication and the seating hours on screen has increased in several folds and the freedom of movement has been reduced considerably. ${ }^{8}$ Lockdown has affected the economy negatively and in a recent interview Nobel laureate Dr Abhijit Banerjee said that "COVID-19 pandemic is far from over and it is uncertain that how long the economy will take to recover".,10 A great magnitude of joblessness and reduced income caused insecurity and uncertainty among people, collectively leading to loneliness; contamination fear; sleep disturbances; worry for loved ones; helplessness,
${ }^{1}$ Department of Physical Education and Sports Sciences, Indira Gandhi Institute of Physical Education and Sports Sciences, University of Delhi, New Delhi, India

${ }^{2}$ Department of Yoga Science, University of Patanjali, Haridwar, Uttarakhand, India

Corresponding Author: Dhananjoy Shaw, Department of Physical Education and Sports Sciences, Indira Gandhi Institute of Physical Education and Sports Sciences, University of Delhi, New Delhi, India, Phone: 011-28544497, +91 8373998064, +91 9013053879, e-mail: dhananjoyshaw1960@gmail.com

How to cite this article: Shaw D, Chand C. Yoga for Improving Mental Health during COVID-19 Pandemic: A Review. East J Psychiatry 2020;23 (1):8-11.

\section{Source of support: Nil}

Conflict of interest: None

boredom; psychological distress such as irritability; frustration, a variety of negative emotions that may progress into long-term symptoms of mental illness such as anxiety, maladjustment, and panic disorder; and depression due to separation from friends, friends and other social activities. , $^{4,10-12}$

Yoga is a nonpharmacological means to develop overall mental health by improving willpower, emotional control, and self-confidence and reducing perceived fatigue, stress, and lifestyle-related disorders due to stress such as diabetes mellitus, hypertension, obesity, and many more through mantras, meditation, pranayama, and asanas. ${ }^{13,14}$ Mindfulness is a technique to purposely focus on the present moment which can be improved with the help of asanas, pranayamas, and meditation. ${ }^{15,16}$ Whereas, mantras are mindfulness chants that helps in improving resilience and in managing stress-related consequences such as insomnia, 
anxiety, hyperarousal, and depression. Studies suggests that yoga, mindfulness, and mantras are an effective alternative to allay fear, distress, intense worry, emotional triggers, and psychological symptoms such as depression and improves self-awareness, empathy, and the spiritual wellbeing as well..$^{15,17-19}$ Therefore, this review will describe the importance and practice of yoga and its positive outcomes for community for promoting positive mental health in the crucial times of novel coronavirus disease outbreak that will be beneficial or a constructive way out.

\section{Methodology}

\section{Search Methodology and Article Selection}

This article is a narrative review of the available literature on coping effects of yoga on mental health relevant to the novel coronavirus disease pandemic. The Preferred Reporting Items for Systematic Review and Meta-analysis (PRISMA) guidelines were followed for this article. The electronic database PubMed was searched using the terms "coronavirus disease," "COVID-19," "yoga," "pranayama," "meditation," and "mindfulness" in multiple permutations and combinations with "mental health" in the time frame ranging from 1 December 2019 to 25 November 2020. A total of 97 studies were recovered, from which 72 results were excluded: 40 because they were repetitive: 23 because they were one of the following: commentary, correspondence, guidelines, short communication, personal account, case report, perspective article, or research proposal; 3 because full-text articles were not available; and 6 studies were excluded because they did not consist the selected keywords. Studies that encompassed stress, anxiety, depression, and its related symptoms affecting mental health exclusively were included. Finally, a total of 24 studies were finalized for the review that were relevant to the concerned topic.

\section{Thematic Analysis of the Selected Articles}

Out of the 24 selected studies, most were research articles $(n=11)$ or review articles $(n=9)$. The rest of the articles were letters to the editor $(n=4)$. All the articles were either directly or indirectly related to mental health during COVID-19 patients and their surrounding members such as nursing staff, doctors, family, and friends. The selection process is described in Figure 1.

\section{Results \\ Yoga and Mental Health of Healthcare Workers during COVID-19 Pandemic}

Due to the rising number of COVID-19 cases there has been an increase in the working hours, the stress of working at high risk in unfamiliar conditions, and social isolation and decrease in leisure time in doctors, nurses, and other healthcare staff who are also referred to as the frontline workers. This has affected their psychological health and resulted in symptoms such as anxiety, depression, increase in work-related stress eventually leading to burnout, fear of infection, insomnia, helplessness, panic, anger, post-traumatic stress disorder (PTSD) and many more and in severe cases may lead to substance abuse or addiction and suicide. ${ }^{5,20-22}$ A study reported that frontline workers in Chinese hospitals show increased prevalence of insomnia, anxiety, depression, and obsessive-compulsive disorder (OCD) than workers in non-healthcare environment. ${ }^{20}$ Most of these symptoms go hand in hand and presence of one may aggravate others. ${ }^{21}$

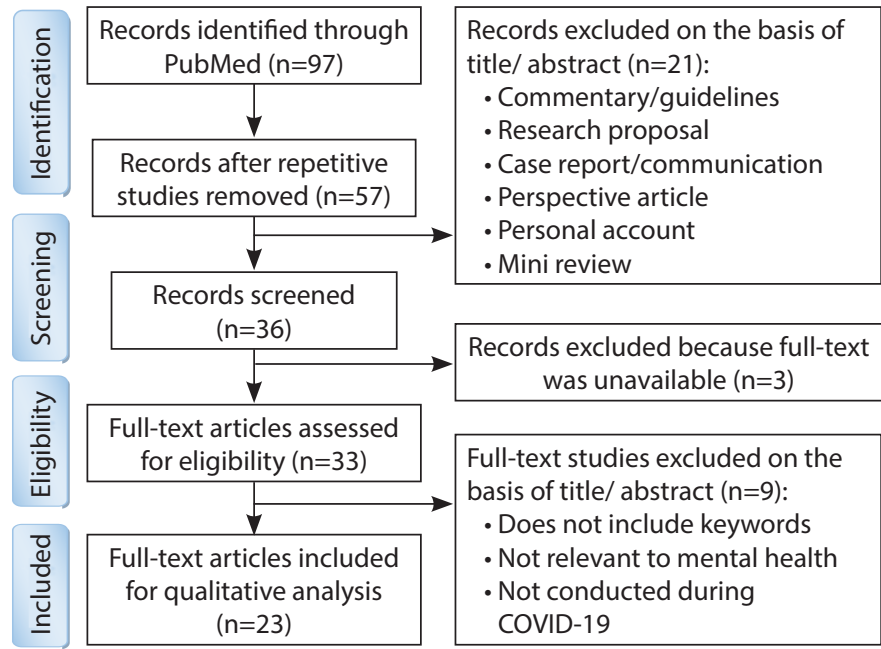

Fig. 1: Flowchart depicting the selection of studies

Along with deliberate positive lifestyle changes such as self-appreciation and persistence to improve the mental well-being of the healthcare staff, muscle relaxation techniques, meditation, mindfulness, and diaphragmatic breathing have been shown to alleviate stress, improve resilience and feeling of connectedness, and reduce the severity of the symptoms. $., 16,20,21,23$

\section{Yoga and Mental Health of Older Adults during COVID-19 Pandemic}

Rapid spread of coronavirus disease and the steps taken to control it has affected the geriatric population and resulted in distress, feelings of worthlessness, and abandonment. It has been found that almost $60-80 \%$ of elderlies suffer from a minimum of one noninfectious condition. ${ }^{24}$ In case of senior citizens due to ageassociated immunosuppression, especially in males suffering with comorbidities such as asthma, diabetes, and hypertension were found to be at a higher risk of infection of COVID-19 with a mortality rate more than $8 \% .{ }^{3,24}$ During the primary stages COVID-19 might cause delirium, depression, anxiety, fatigue, and PTSD. ${ }^{3}$

Yoga being a mind-body medicine has been found to improve the physical aspects and mental parameters in older adults by elevating positive mood states, promoting better sleep quality, and reducing anxiety and depression that can eventually reduce the chances of infection by positive regulation of the immune system Yoga comprising breath regulation, meditation, postures, and mindfulness are safe, affordable, and easy to practice specially for older adults suffering with comorbidities. ${ }^{24}$ Some studies indicate that in older adults, yoga is superior to common physical activity intervention. ${ }^{25}$

\section{Online Management of Mental Health through Yoga during COVID-19}

It has been evident from recent studies that COVID-19 can cause negative impact on mental health of all age-groups and the symptoms can be short-term or long-term and yoga and mindfulness can improve the overall wellbeing of an individual during and after the coronavirus disease pandemic and due to geographic limitations online mode of communication has become a boon. ${ }^{26,27}$ A preliminary study of 4 weeks conducted by Jasti et al. revealed that online yoga sessions can be safe and useful in reducing stress, improving psychological relaxation, calmness, and concentration..$^{26}$ Another study performed by Drissi et al. to the 
working capabilities of highly rated anxiety applications on mobile phones showed that 147 applications used meditation, breathing exercises, yoga, mindfulness, and guided relaxation to cope with anxiety and related disorders such as stress, depression, eating and sleep disorders, and many more. ${ }^{28}$

However, El Morr et al. depicted that virtual sessions of mindfulness were useful in reducing depression and anxiety but were ineffective in reducing perceived stress in college students. ${ }^{27}$ Due to constant exposure of young adults to malinformation on social media platforms worsening of psychological symptoms were observed and it was revealed that mindfulness acts as a preventive measure to reduce distress caused by social media. ${ }^{29}$

\section{Discussion}

The fundamental medicine principle "Prevention is better than cure" must be followed by everyone to reduce the cases of infection. The most common psychological symptoms observed during the pandemic were stress, anxiety, and depression. Some simple and useful home-based yoga and meditation practices can be used as a preventive measure and also as recovery management of COVID-19. ${ }^{7,30}$ Weilgus et al. indicated by using path analysis that there exists a positive relationship between anxiety and psychological responses to the pandemic and mindfulness decreased the level of anxiety. ${ }^{31}$ Similar results were obtained by Brose et al. when they showed that increase in mindfulness resulted in decrease in negative mental health symptoms and vice versa. ${ }^{11}$ Studies has shown that mantras, a spiritual coping mechanism are useful in stress management for people suffering with PTSD and chronic diseases and for healthcare workers as well. ${ }^{17}$

Since SAR- CoV-2 is a heat-sensitive enveloped virus therefore, jala neti and ghrita neti or the nasal oil application using lukewarm water and oil, respectively are efficient in upper respiratory tract infections and has been proposed as a preventive method by Traditional Chinese Medicine researchers as it forms a biofilm and prevents the entry of virus. ${ }^{7,32}$ Heat-based therapies such as steam has been found to improve physical and mental relaxation, sleep, and mindfulness. ${ }^{33}$

Tele-yoga sessions and mobile applications can be used to decrease the adverse effects of sedentary lifestyle. ${ }^{25,28}$ Most of the studies included in the review had limitations due to small sample size and subjective nature of the study. However, since the COVID-19 pandemic is persisting and new strains are emerging leading to lockdown again in many countries, poor mental health will continue even when specific treatment protocol and vaccination will be available in due course of time; therefore, there is a need of lot of research to understand the post effect of COVID-19 after recovery.

\section{Conclusion}

The practices of yoga and mindfulness will be of great benefit even during the post-COVID-19 era. It was concluded that yoga and mindfulness can be used as a coping mechanism to improve mental health, emotional control, self-confidence, concentration, motivation, and quality of life. ${ }^{2,4,14}$ It reduces stress, negative thoughts, anxiety, panic, and also helps in the management of sleep, depression, and feeling of loneliness. ${ }^{1,4,6,12,25}$ Thereby, yoga helps in calming, soothing, and relaxing the mind and body of the affected patients, families, pregnant females, police personnel, care staff, and medical and paramedical staff during and after the pandemic. $^{1,12,23,25,33}$

\section{References}

1. Ahadi MS, Sahraian MA, Rezaeimanesh N, et al. Psychiatric advice during covid-19 pandemic for patients with multiple sclerosis. Iran J Psychiatry Behav Sci 2020;14:103243. DOI: 10.5812/ijpbs.103243

2. Sun $N$, Wei $L$, Shi S, et al. A qualitative study on the psychological experience of caregivers of COVID-19 patients. Am J Infect Control 2020;48:592-598. DOI: 10.1016/j.ajic.2020.03.018

3. Jiménez Ó, Sánchez-Sánchez LC, García-Montes JM. Psychological impact of COVID-19 confinement and Its relationship with meditation. Int J Environ Res Public Health 2020;17:6642. DOI: 10.3390/ ijerph17186642

4. Hiremath P, Suhas Kowshik CS, Manjunath M, et al. COVID 19: impact of lock-down on mental health and tips to overcome. Asian J Psychiatr 2020;51:102088. DOI: 10.1016/j.ajp.2020.102088

5. Bansal $P$, Bingemann TA, Greenhawt $M$, et al. Clinician wellness during the COVID-19 pandemic: extraordinary times and unusual challenges for the allergist/immunologist. J Allergy Clin Immunol Pract 2020;8:1781-1790. DOI: 10.1016/j.jaip.2020.04.001

6. Mukhtar S. Psychological health during the coronavirus disease 2019 pandemic outbreak. Int J Soc Psychiatry 2020;66:512-516. DOI: $10.1177 / 0020764020925835$

7. Tillu G, Chaturvedi S, Chopra A, Patwardhan B. Public health approach of ayurveda and yoga for COVID-19 prophylaxis. J Altern Complement Med 2020;26:360-364. DOI: 10.1089/acm.2020.0129

8. Toniolo-Barrios M, Pitt L. Mindfulness and the challenges of working from home in times of crisis. Bus Horiz 64(2):189-197. DOI: 10.1016/j. bushor.2020.09.004

9. Hindustan Times [Internet]. HTLS 2020: No guarantee that we're out of Covid-19 pandemic, says Abhijit Banerjee [updated 2020 Dec 10; cited 2020 Dec 24]. Available from: https://www.hindustantimes.com

10. Statista Research Department [Internet]. COVID-19 impact on unemployment rate in India 2020 [updated 2020 Dec 7; cited 2020 Dec 24]. Available from: www.statista.com

11. Brose A, Blanke ES, Schmiedek F, et al. Change in mental health symptoms during the COVID-19 pandemic: the role of appraisals and daily life experiences. J Pers 2020.

12. Chevance A, Gourion D, Hoertel N, et al. Ensuring mental health care during the SARS-CoV-2 epidemic in France: a narrative review. Encephale 2020;46:193-201. DOI: 10.1016/j.encep.2020.04.005.

13. Nagarathna R, Nagendra HR, Majumdar V. A perspective on yoga as a preventive strategy for coronavirus disease 2019. Int J Yoga. 2020;13:89-98. DOI: 10.4103/ijoy.IJOY_22_20

14. RansingR,PintodaCostaM,AdiukwuF,etal.YogaforCOVID-19andnatural disaster related mental health issues: challenges and perspectives. Asian J Psychiatr 2020;53:102386. DOI: 10.1016/j.ajp.2020.102386

15. Gaiswinkler L, Unterrainer HF. The relationship between yoga involvement, mindfulness and psychological well-being. Complement Ther Med 2016;26:123-127. DOI: 10.1016/j.ctim.2016.03.011

16. Rana W, Mukhtar S, Mukhtar S, et al.Psychological health of aging mental healthcare social workforce amidst coronavirus disease-2019 pandemic. Int J Geriatr Psychiatry 2020;36(3):461-462.

17. Oman D, Bormann JE, Kane JJ. Mantram repetition as a portable mindfulness practice: applications during the COVID-19 pandemic. Mindfulness (N Y) 2020; 1-12.

18. Matiz A, Fabbro F, Paschetto A, et al. Positive ilmpact of mindfulness meditation on mental health of female teachers during the COVID-19 outbreak in Italy. Int J Environ Res Public Health 2020; 17: 6450. DOI: 10.3390/ijerph17186450

19. Baiano C, Zappullo I, The LabNPEE Group, et al. Tendency to worry and fear of mental health during Italy's COVID-19 lockdown. Int J Environ Res Public Health 2020;17:5928. DOI: 10.3390/ ijerph17165928 
20. Chow KM, Law BMH, Ng MSN, et al.A review of psychological issues among patients and healthcare staff during two major coronavirus disease outbreaks in China: contributory factors and management strategies. Int J Environ Res Public Health 2020;17:6673. DOI: 10.3390/ ijerph17186673

21. Balasubramanian A, Paleri V, Bennett R, et al. Impact of COVID-19 on the mental health of surgeons and coping strategies. Head Neck 2020;42(7):1638-1644. DOI: 10.1002/hed.26291

22. Magnavita N, Soave PM, Ricciardi W, et al. Occupational stress and mental health among anesthetists during the COVID-19 pandemic. Int J Environ Res Public Health 2020;17:8245. DOI: 10.3390/ijerph17218245

23. DeyA,MajumdarP, Saha A, etal.COVID-19pandemiclockdown-induced altered sleep/wake circadian rhythm, health complaints and stress among traffic police personnel in India 2000;38(1):140-148. DOI: 10.1080/07420528.2020.1831524.

24. Mohanty S, Sharma P, Sharma G. Yoga for infirmity in geriatric population amidst COVID-19 pandemic: comment on "Age and ageism in COVID-19: Elderly mental health-care vulnerabilities and needs". Asian J Psychiatr 2020;53:102199.

25. Banskota S, Healy M, Goldberg EM. 15 smartphone apps for older adults to use while in isolation during the COVID-19 pandemic. West J Emerg Med 2020; 21: 514-525. DOI: 10.5811/westjem.2020.4.47372

26. Jasti N, Bhargav H, George S, et al. Tele-yoga for stress management: need of the hour during the COVID-19 pandemic and beyond? Asian J Psychiatr 2020;54:102334.

27. El Morr C, Ritvo P, Ahmad F, et al. Effectiveness of an 8-week web-based mindfulness virtual community intervention for university students on symptoms of stress, anxiety, and depression: randomized controlled trial. JMIR Ment Health 2020;7:e18595. DOI: $10.2196 / 18595$

28. Drissi N, Ouhbi S, Janati ldrissi MA, et al. An analysis on self-management and treatment-related functionality and characteristics of highly rated anxiety apps. Int J Med Inform 2020;141:104243.20. DOI: 10.1016/j.ijmedinf.2020.104243

29. Hong W, Liu RD, Ding Y, et al. Social media exposure and college students' mental health during the outbreak of COVID-19: the mediating role of rumination and the moderating role of mindfulness. Cyberpsychol Behav Soc Netw 2021;24(4):282-287

30. Makhashvili N, Javakhishvili JD, Sturua L, et al. The influence of concern about COVID-19 on mental health in the Republic of Georgia: a cross-sectional study. Global Health 2020;16:111. DOI: 10.1186/ s12992-020-00641-9

31. Wielgus B, Urban W, Patriak A, et al. Examining the associations between psychological flexibility, mindfulness, psychosomatic functioning, and anxiety during the COVID-19 pandemic: a path analysis. Int J Environ Res Public Health 2020;17:8764. DOI: 10.3390/ ijerph17238764

32. Cohen M. Turning up the heat on COVID-19: heat as a therapeutic intervention. F1000Res 2020;9:292. DOI: 10.12688/ f1000research.23299.2

33. Traylor CS, Johnson JD, Kimmel MC, et al. Effects of psychological stress on adverse pregnancy outcomes and nonpharmacologic approaches for reduction: an expert review. Am J Obstet Gynecol MFM 2020;2:100229. DOI: 10.1016/j.ajogmf.2020.100229 